\title{
NEGOTIATING THE SELLING PRICE OF HYDROPOWER ENERGY USING MULTI-AGENT SYSTEMS IN BOT
}

\author{
Emre Caner AKCAY ${ }^{1^{*}}$, Irem DIKMEN ${ }^{2}$, M. Talat BIRGONUL ${ }^{2}$, David ARDITI ${ }^{1}$ \\ ${ }^{1}$ Department of Civil, Architectural and Environmental Engineering, Illinois Institute of Technology, \\ Alumni Memorial Hall, 3201 S. Dearborn St., 60616, Chicago, USA \\ ${ }^{2}$ Department of Civil Engineering, Middle East Technical University, Dumlupinar Bulvari-1, \\ 06800 Ankara, Turkey
}

Received 05 November 2018; accepted 14 March 2019

\begin{abstract}
During the feasibility study of BOT (Build-Operate-Transfer) hydropower investments, the selling price of energy is the most critical parameter that impacts the net present value (NPV) estimated by the investors. Investors usually consider the price of energy guaranteed by the government during their feasibility studies which is the worst case scenario. However, it is apparent that negotiations that take place between investor and broker determine the price of energy which is affected by various sources of uncertainty associated with the energy demand and country conditions. The objective of this study was to make a realistic estimate of the investor's selling price by modeling the negotiation process between investor and broker using a multi-agent system (MAS). Thus, the factors affecting the negotiation process were identified, a negotiation protocol between the parties was set up, negotiation scenarios were determined, and modelled by using a MAS. The model was tested on a hydropower investment in Turkey and generated more realistic results compared to the current practice. Investors and brokers may benefit from this study because it considers the potential changes in the market as well as the negotiating postures of parties under different scenarios.
\end{abstract}

Keywords: renewable energy, hydropower investment, multi-agent system, negotiation.

\section{Introduction}

A Public Private Partnership (PPP) is a contractual agreement between a public agency and a private entity for financing, designing, constructing, and operating infrastructure facilities (Zayed \& Chang, 2002). PPP can take several forms such as Design-Bid-Build (DBB), Private Contract Fee Services, Design-Build (DB), Build-OperateTransfer (BOT), Long Term Lease Agreements, DesignBuild-Finance-Operate (DBFO), and Build-Own-Operate (BOO).

The BOT system is especially useful in developing countries where governments cannot finance infrastructure projects due to lack of funds (Shen \& Wu, 2005). Developing countries often use BOT to invest in hydropower plants, whereby a private entity makes an investment with their own resources or with bank credit, and then makes loan repayments by selling energy over a concession period agreed upon by the government and the private entity (J. Song, D. Song, \& Zhang, 2015). According to a questionnaire survey administered to 50 experts with ex- perience in hydropower plant investments for more than 20 years, the selling price of energy is the most critical parameter that impacts the estimated net present value (NPV) of such a project undertaken using BOT (Akcay, Dikmen, Birgonul, \& Arditi, 2017). Indeed, during the concession period, the selling price of energy should be as close as possible to or greater than the estimated selling price considered in the feasibility study. The selling price of energy is difficult to predict due to the existence of various sources of uncertainty about the energy market. In general, the minimum selling price of energy is guaranteed by the government. Investors typically use this guaranteed price in their feasibility studies. However, in practice, investors do not sell the energy to the government. They prefer to sell the energy to companies that act as brokers because brokers pay them a higher price than the price guaranteed by the government. Brokers buy the energy from investors and sell this energy to consumers such as factories, hotels, and hospitals. The selling price of

${ }^{*}$ Corresponding author. E-mail: eakcay@iit.edu 
energy is determined as a result of a negotiation between investors and brokers. The objective of this research is to estimate a realistic revenue stream by modeling the negotiation process between an investor and a broker using a multi-agent system.

\section{Multi-agent systems}

A multi-agent system (MAS) consists of agents who are defined as multiple interacting intelligent elements within an environment. The agents have two important attributes. First, they enjoy autonomous actions. Shoham (1993) describes autonomous actions as actions that are performed without constant human guidance. In other words, agents behave according to the assigned design objectives. The second attribute of agents is a capability to interact with other agents in the system. Kraus, Wilkenfeld, and Zlotkin (1995) state that agents can have different goals and targets and can be in real competition with each other. Real life negotiations can be modelled by imitating agent interactions. According to Kraus et al. (1995), agents are motivated by goals that allow them to achieve mutually acceptable solutions. As Cleary (2001) stated, negotiation is a costly and time consuming process. It is important to find a solution that is acceptable to all parties. Some negotiation protocols simulate real life negotiations and are extensively used in MAS (Karakas, 2010). Kraus et al. (1995) describe a negotiation protocol as a mechanism that is used for dealing with issues faced by agents. There are two main negotiation protocols that are commonly used. These are the Zeuthen Strategy and the Zeuthen Strategy with Bayesian Learning. In the Zeuthen Strategy, the agents are fully informed of each other's position, whereas in the Zeuthen strategy with Bayesian learning, they are not.

Agent-based modelling is used to derive findings for a system's behavior (macro level) using the agents' behavior (micro level). It can be used for predicting consequences, performing tasks (such as planning, resource and allocation, etc.), or discovering theory. Computational experimentation by means of simulation is carried out using agent-based models. Agent-based modelling and simulation provide new options to address the challenges of planning and prediction in social systems. Starting from the 1980s it has been widely used in many disciplines such as management sciences, economics, sociology, systems engineering, computer science, and biology as an approach to gain theoretical insights into complex systems. However, their use in the construction industry has only recently gained the attention of researchers. Ren and Anumba (2004) explored the use of multi-agent systems in the construction industry and discussed their advantages. Using multi-agent systems, De Oliveira, Fonseca, and Steiger-Garcao (1997) developed a model that manages resources in a construction company. Tah (2005), Xue, Li, Shen, and Wang (2005), Xue, Shen, O'Brien, and Ren (2009) generated a modelling and simulation platform for use in construction supply chain management. Kim and Paulson (2003) proposed a negotiation model to facilitate the distributed coordination of project schedule changes; Taylor, Levitt, and Villarroel (2009) proposed a simulation model to discover the effects of learning dynamics in project networks; $\mathrm{Ng}$ and Li (2006) developed automated negotiation for the sourcing of construction suppliers; Molinero and Núñez (2011) tested a model that plans work schedules during the construction of a building. Taghaddos, Hermann, AbouRizk, and Mohamed (2014) performed an agent-based simulation model to schedule modular construction. Karakas, Dikmen, and Birgonul (2013) proposed a model that simulates the negotiation process between client and contractor for sharing of risk and cost overruns in construction projects. El-Adaway and Kandil (2010) developed a model that can resolve construction disputes. Du and El-Gafy (2012) proposed an agent-based model to investigate the interactions of organizational and human factors on construction performance. Most recently, Mostafavi, Abraham, DeLaurentis, Sinfield, Kandil, and Queiroz (2015) developed an agent-based model to simulate the dynamics of highway transportation infrastructure financing. González-Briones, Chamoso, De La Prieta, Demazeau, and Corchado (2018) presented an agent based model to optimize the use of energy; Zhu, Zhao, and Chua (2016) prepared an agent-based debt terms' bargaining model to simulate the negotiation process in PPP projects. Farshchian, Heravi, and AbouRizk (2017) presented an agent-based simulation model to simulate budget allocation and its effects on projects' progress in an owner's portfolio of construction projects, and González-Briones, Prieto, De La Prieta, Herrera-Viedma, and Corchado (2018) developed an energy saving system which takes decisions to optimize energy consumption by using a multi-agent system.

It is clear that multi-agent systems have been used over the years in the construction management literature for several purposes (e.g., resource management, scheduling, budget allocation, supply chain management, dispute resolution, etc.), but have not been used for the prediction of energy price in PPP projects yet. In this study, a MAS has been developed to simulate the negotiation process between an investor and a broker and to predict the selling price of energy under different scenarios. The system has been developed considering the dynamics of the Turkish energy market which will be briefly explained in the next section.

\section{BOT in the Turkish energy sector}

The PPP model was first coined in Turkey in 1984 to solve the energy bottleneck (Ozdoganm \& Birgonul, 2000). However, the BOT model could not be used effectively due to legal uncertainties and lack of government guarantees that increased the risk carried by investors. In 2001, the government provided a legal basis for investors to produce and sell energy in the energy market with Law No. 4628. 
Later, in 2005, the government announced the renewable energy regulations and Law No. 5346, which provide a guarantee to investors that energy will be purchased by the government. These laws encouraged investors to invest in renewable power plants using the PPP system. As a result, 575 hydropower projects, amounting to $\$ 6.5$ billion, have been tendered on a PPP basis in Turkey since 2005 (Akcay et al., 2017).

\section{Development of a multi-agent system for the prediction of the selling price of hydropower energy}

The objective of this study is to develop a multi-agent system to simulate the negotiation between an investor and a broker to agree on the selling price of energy. Scenarios are set up considering the risk factors involved in such projects, and the energy price is predicted in each of the different scenarios. The predicted values for each scenario are used in the feasibility studies conducted by the investors. The development of a multi-agent system involves several stages. First, the interaction and negotiation process between the investor and the broker should be understood and modelled. The proposed generic framework for the development of the system is presented in Figure 1. Then, the risk factors, particularly the sources of uncertainty that may lead to changes in energy prices and in the position/ power of the parties to the negotiations have to be as- sessed. In this study, to elicit this information, a questionnaire survey was administered to a representative sample of investors and brokers, as explained in the next section.

A negotiation process has to be defined between the investor and the broker to determine the selling price of energy. To start the negotiation process, not only are the first offers of the agents considered, but also their reservation values need to be specified. The reservation values show the limits for each agent. For the investor, it is the lowest price that can be accepted for the energy sold. The investor never accepts any price that is lower than the investor's reservation value. For the broker, the reservation value is the highest price that can be paid to the investor. The broker never offers a higher price than the broker's reservation value.

The investor makes the first offer to the broker. The purpose is to sell the energy at a price that is as high as possible as, certainly higher than the price guaranteed by the government; this is the investor's "reservation value". The investor's first offer is made by considering the risk factors prevailing in the market. The broker makes a counteroffer to the investor. In contrast to the investor, the broker's ultimate purpose is to buy the energy at the lowest possible price, certainly lower than the broker's selling price to customers, called the broker's "reservation value". The broker also considers the risk factors. As per Karakas' et al. (2013) recommendation, the broker's first counteroffer is typically $70 \%$ of the broker's reservation value.

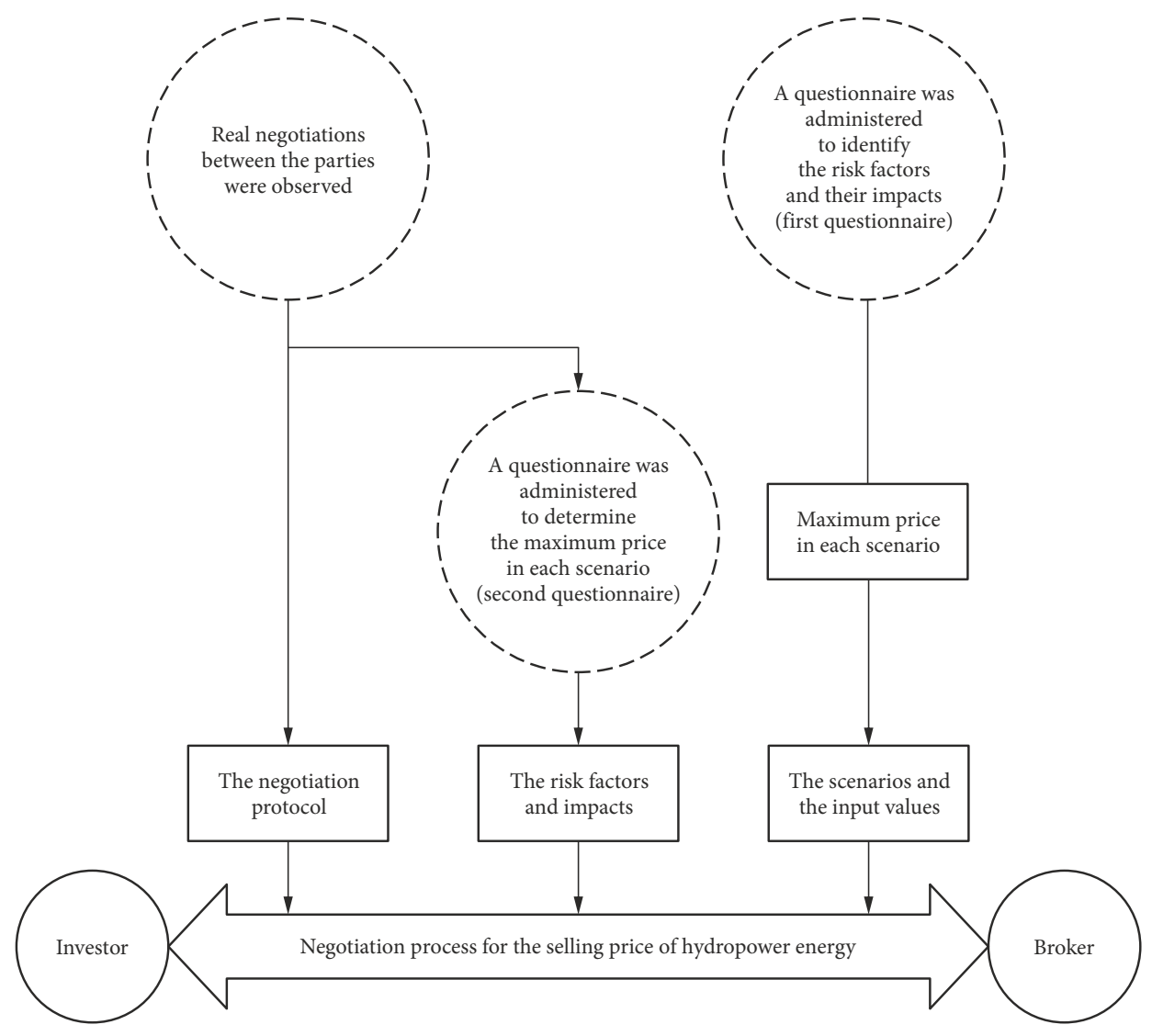

Figure 1. Proposed multi-agent system for the negotiation of the selling price of energy 
The next step is to set up a negotiation protocol. The Zeuthen strategy is identified as the most appropriate negotiation strategy, because fully informed agents know the risk factors and they are aware of each other's reservation values. As Karakas et al. (2013) stated, in the Zeuthen Strategy, the negotiation process is simulated by comparing losses and gains, which means that for each proposal, the investor and the broker calculate the utility of their loss/gain. In this negotiation process, as recommended by Karakas et al. (2013), the utility of the investor's and of the broker's first offers is set to " 1 " and the utility of their reservation values is set to 0.6 . The utility of each intermediate offer is calculated by linear interpolation between these values.

The negotiation process involves competitive pressures that manifest themselves as a desire to agree as quickly as possible. For example, if the investor delays the negotiations, the produced energy will have to be sold to the government at the guaranteed price, which is actually the worst case scenario. On the other hand, if the broker delays the negotiations, opportunities to sell energy to factories, hospitals, and other clients will be lost. Every offer/ counteroffer round reduces the utility by $10 \%$. Based on this negotiation protocol, the proposed multi-agent system was implemented in a real hydropower project which will be described in detail in the following section.

\section{Application to a real hydropower project}

A small hdyropower project located in Turkey was chosen as a case study. This project had four successive stages, each stage adding 7.5 MW to the capacity. According to the pre-feasibility study, this hydroelectric power plant was planned to work 3,000 hours per year.

To model the negotiation process between investor and broker using a multi-agent system, more than 50 negotiations were observed. The projects considered were located in different regions of Turkey, allowing the researchers to understand the dynamics of the negotiation process in each region, and as a result, construct a comprehensive model for the negotiation. To identify the risk factors and their impacts during the negotiation process, a questionnaire of two parts was administered to 10 brokers and 30 investors. In the first part of the questionnaire, respondents were asked to identify the risk factors affecting the energy price during negotiations. In the second part, respondents rated the impact of these risk factors. 30 investors were chosen at random from the members of the Turkish Contractors Association which represents the leading construction companies in Turkey (Turkish Contractors Association [TCA], 2017). The business volume of its members amounts to almost $70 \%$ of all domestic and $90 \%$ of all international contracting work performed by Turkish construction companies (TCA, 2017). In order to obtain consistent survey results, the investors who participated in the survey had hydropower investments in at least one of Turkey's seven regions. The 10 brokers had previously worked with the 30 investors who participated in the study. The participants stated five risk factors in response to the first part of the questionnaire survey. The risk factors are identified as the sources of uncertainty affecting the selling price of energy and consequently, the power of the parties to the negotiations.

- The first risk factor is energy demand. If energy demand is low, there are few customers to whom the investor can sell the produced energy, or there are many customers but their energy needs are stagnant. In this case, the broker is more powerful during negotiations. On the other hand, if the energy demand is high, the investor has the negotiating power.

- The second risk factor is the level of competition defined by the number of competitors that produce energy. If there are few competitors, the investor has the power during negotiations whereas if the level of competition is high, the broker has the power.

- The third risk factor is the production capacity of the hydroelectric power plant. If the investor possesses a hydroelectric power plant that has a large energy production capacity, the investor can meet the demand regardless of how high demand is. As a result of this condition, the investor is in a better position to negotiate. On the other hand, if the investor's hydroelectric power plant has a low capacity; the broker has the power in the negotiation.

- The fourth risk factor is the economic conditions prevailing in the country. In this case, it is assumed that neither party has an advantage over the other regardless of how favorable or unfavorable the economic conditions are. It is worth noting however that when economic conditions are good, the first offers of both parties are higher compared to the offers that would be made when economic conditions are poor.

- The fifth risk factor involves changes in the laws. It is assumed that regardless of whether many or no changes will be made in the laws over the years, investors and brokers equally share the negotiation power since it is not possible to predict whether the changes will provide any benefit to the investor or to the broker. It should also be noted that when no changes are predicted, the first offers of both parties are higher compared to the offers under unstable conditions where many changes are expected.

The same respondents rated the impact of these risk factors using a 10-point scale, later converted to \% values that add up to 1 . The modes of the ratings that have been used in subsequent analysis are presented in Table 1. However, it is clear that due to the vagueness and the existence of epistemic uncertainty associated with the risk values, a level of fuzziness should also be incorporated into the analysis. Respondents assessed the level of vagueness as low, moderate and high (Table 1) because it is easier for them to make this assessment linguistically. Later, these values were converted into percentage values based on subjective judgements. 
Table 1. Modes of risk factors' impacts assessed by the respondents

\begin{tabular}{|l|c|c|c|}
\hline \multicolumn{1}{|c|}{ Risk Factor } & Mode of impact (Out of 10) & Percent impact (\%) & Fuzziness level \\
\hline Demand & 10 & $35.7 \%$ & Low \\
\hline Level of competition & 6 & $21.4 \%$ & Moderate \\
\hline Production capacity & 7 & $25.0 \%$ & Low \\
\hline Economic conditions & 3 & $10.7 \%$ & High \\
\hline Changes in the laws & 2 & $7.1 \%$ & Low \\
\hline Total & 28 & $100 \%$ & \\
\hline
\end{tabular}

The scenarios used in this study are generated considering the varying impacts of risk factors. An example scenario is presented in Figure 2. In the example scenario, the reservation values and the first offers of the parties are expressed in percentages. These percentages represent a value between the minimum and maximum energy prices. The minimum price is taken as $6 \mathrm{~d} / \mathrm{kWh}$ which is the price guaranteed by the Turkish government. This minimum price is always constant and corresponds to " $0 \%$ " while the maximum price corresponds to "100\%" in the interpolation. In order to determine the maximum price in each scenario, a questionnaire survey of two parts was administered to the 10 brokers and 30 investors who previously determined the risk factors and their impacts. In the first part, all risk factors were presented to them, and they were asked to identify those that affect the maximum price. The consensus was that "economic conditions" and "changes in laws" are the risk factors that most affect the maximum price. The second part of the questionnaire sought the maximum price, considering these two risk factors. As each risk factor has two alternative states, there are four different scenarios related to maximum price. So in the second part of the questionnaire, these four possible scenarios were presented to the respondents, and the maximum price for each scenario was solicited. The means of the respondents' answers are presented in Table 2. The next step is to convert the percentage values (\%) to prices $(\$ / \mathrm{kWh})$ for the first offers and reservation values in each scenario, in order to start the negotiation process in the proposed multi-agent system.
Table 2. The maximum prices for different scenarios

\begin{tabular}{|l|l|c|}
\hline $\begin{array}{c}\text { Economic } \\
\text { conditions }\end{array}$ & \multicolumn{1}{|c|}{ Changes in laws } & $\begin{array}{c}\text { Maximum price } \\
(\$ / \mathrm{kWh})\end{array}$ \\
\hline Favourable & No changes expected & 20 \\
\hline Favourable & Adverse changes expected & 19 \\
\hline Unfavourable & No changes expected & 18 \\
\hline Unfavourable & Adverse changes expected & 17 \\
\hline
\end{tabular}

For the investor's first offer in the example scenario (Figure 2) is as follows. The investor's first offer expressed in percentage was determined by adding the risk factors on which the investor has power $(41.1 \%)$ and the risk factors that the investor shares with others (22.6\%), as shown in Figure 2.

First offer of investor expressed in

percentage $=41.1 \%+22.6 \%$

$$
=63.70 \% \text {; }
$$

Minimum price $=6 \mathrm{\Phi} / \mathrm{kWh}$ (for all scenarios);

Maximum price $=20 \mathrm{\$} / \mathrm{kWh}$ (from Table 2 for good economic conditions and no changes in laws predicted in the example scenario).

Using Eqn (1), the corresponding price for the investor's first offer of $63.70 \%$ is calculated by interpolating between the guaranteed minimum price $(6 \mathrm{c} / \mathrm{kWh})$ and the maximum price $(20 \mathrm{\$} / \mathrm{kWh})$ :

$$
=>6+\left[(20-6) \cdot \frac{63.7}{100}\right]=14.9 \$ / \mathrm{kWh} .
$$

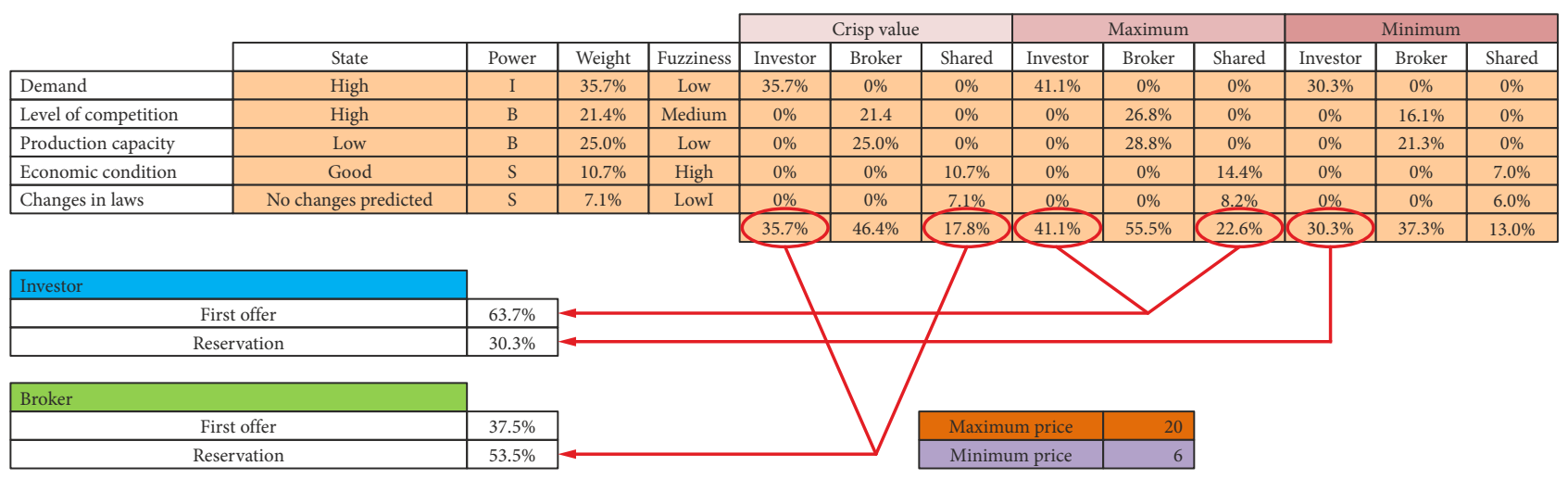

Figure 2. An example scenario 
The percentage values for the first offers and reservation values presented in Table 2 were converted to "prices" for all scenarios, by interpolation. The number of possible scenarios in the negotiation process is calculated as 32 considering the 5 risk factors and their alternative states.

Using a multi-agent system with the Zeuthen Strategy, the selling price of energy is calculated for these 32 scenarios, and 32 different results are obtained at the end of the simulation. In each scenario, once the first offers and the reservation values are obtained from the investor and the broker, the multi-agent system performs the negotiation process between investor and broker using the Zeuthen Strategy and leading to an outcome that is acceptable to both agents. The number of negotiation rounds varies in each scenario. All 32 scenarios were defined in a computerized system that uses a Java Agent Development (JADE) platform, and negotiations were simulated

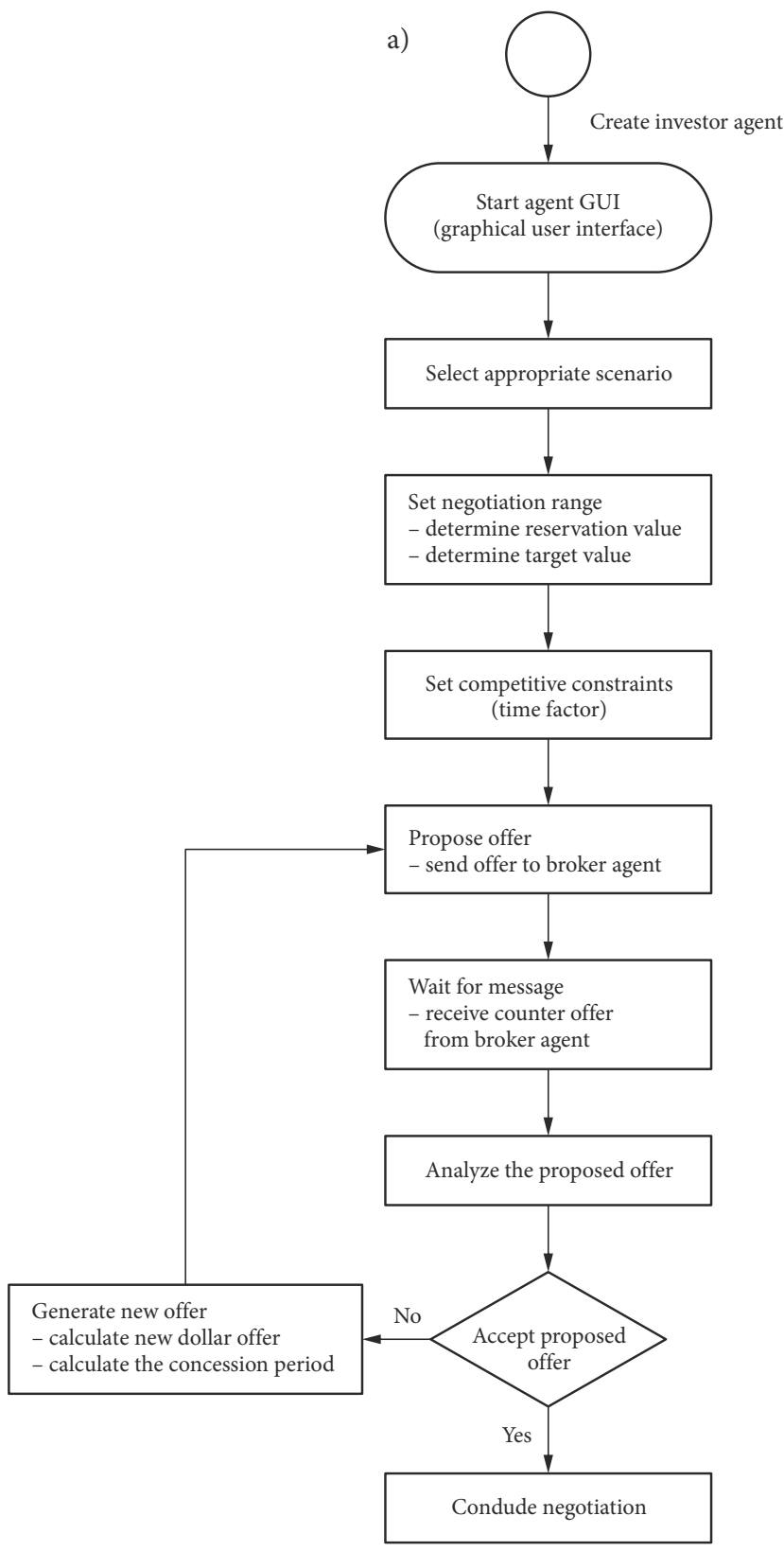

between the two agents (Broker and Investor). The agents' state-of-mind, demands, and objectives are shown in detail in Figure 3. In addition, the interaction between two agents in the negotiation process is shown in Figure 4. The overall duration of the simulation for 32 scenarios is 5-6 minutes. The outcomes of the negotiations are presented in Table 3.

As shown in Figure 4, first of all, the user chooses the appropriate scenario by assessing the risk factors (e.g., energy demand is high or low) based on their expectations about the future. Each agent draws the borders for the negotiation process based on their negotiation zone which shows the interval between their first offer and reservation value. In order to take into account the time pressure during negotiations, a value (10\%) is specified within the model and then the negotiation starts. After several negotiation rounds, the settlement amount is determined.

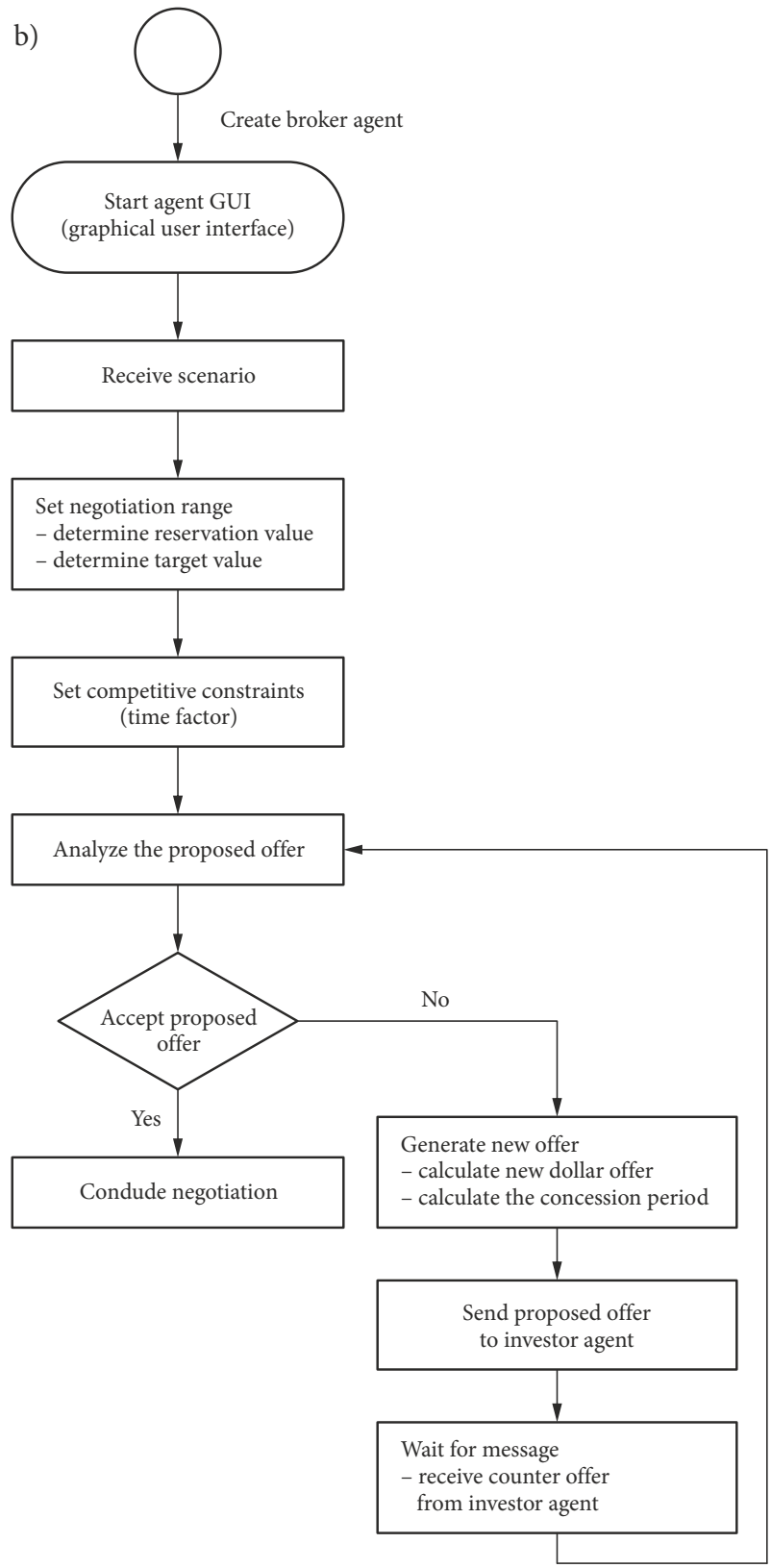

Figure 3. (a) State diagram for investor's behavior; (b) State diagram for broker's behavior 
Table 3. Negotiation results for each scenario

End of Table 3

\begin{tabular}{|l|c|}
\hline \multicolumn{1}{|c|}{ Scenarios } & Selling price of energy $(\mathbb{\Phi} / \mathrm{kWh})$ \\
\hline Scenario-1 & 11.84 \\
\hline Scenario-2 & 11.42 \\
\hline Scenario-3 & 10.97 \\
\hline Scenario-4 & 10.59 \\
\hline Scenario-5 & 14.54 \\
\hline Scenario-6 & 13.85 \\
\hline Scenario-7 & 13.32 \\
\hline Scenario-8 & 12.65 \\
\hline Scenario-9 & 15.29 \\
\hline Scenario-10 & 14.64 \\
\hline Scenario-11 & 13.95 \\
\hline Scenario-12 & 13.30 \\
\hline Scenario-13 & 18.02 \\
\hline Scenario-14 & 17.15 \\
\hline Scenario-15 & 16.28 \\
\hline Scenario-16 & 15.4 \\
\hline
\end{tabular}

\begin{tabular}{|l|c|}
\hline \multicolumn{1}{|c|}{ Scenarios } & Selling price of energy $(\mathbb{\$} / \mathrm{kWh})$ \\
\hline Scenario-17 & 13.05 \\
\hline Scenario-18 & 12.50 \\
\hline Scenario-19 & 12.05 \\
\hline Scenario-20 & 11.58 \\
\hline Scenario-21 & 9.80 \\
\hline Scenario-22 & 9.60 \\
\hline Scenario-23 & 9.30 \\
\hline Scenario-24 & 9.00 \\
\hline Scenario-25 & 10.44 \\
\hline Scenario-26 & 10.15 \\
\hline Scenario-27 & 9.78 \\
\hline Scenario-28 & 9.44 \\
\hline Scenario-29 & 7.70 \\
\hline Scenario-30 & 7.60 \\
\hline Scenario-31 & 7.50 \\
\hline Scenario-32 & 7.40 \\
\hline
\end{tabular}

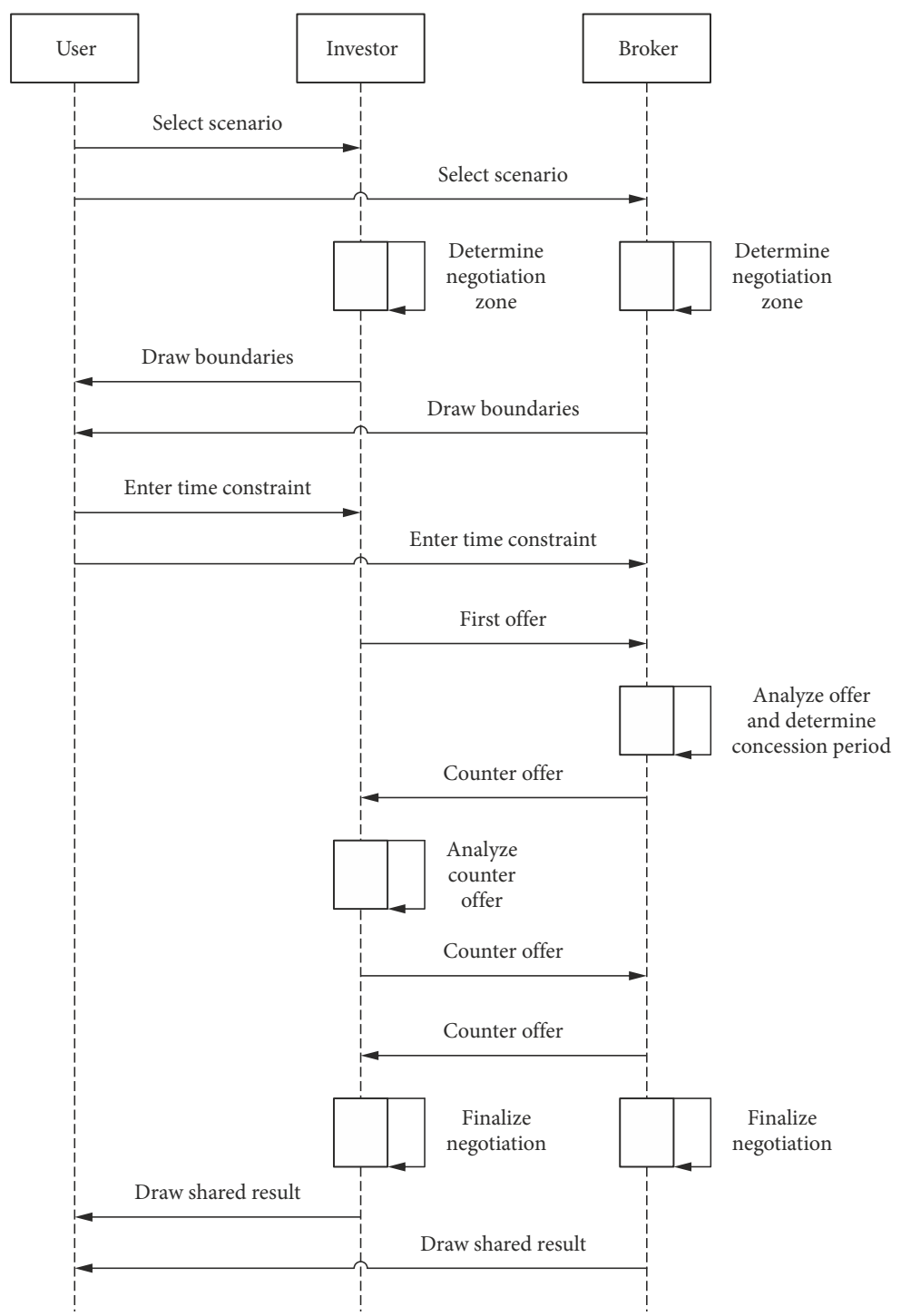

Figure 4. Sequence diagram for user and agents 


\section{Discussion of findings}

In this section, the use of the findings of the proposed MAS is discussed in performing the feasibility study of a hydropower project. In the project used as a case study, if the selling price of the energy is assumed to be equal to the price guaranteed by the government $(6 \mathrm{c} / \mathrm{kWh})$, the net present value (NPV) of the project would be $\$ 27,845,376.65$. If the selling price of energy is considered to be the average of the selling prices obtained in the 32 scenarios that reflect the supply-demand conditions and the negotiations between parties, the net present value is found as $\$ 66,475,096.17$. Significant differences can be seen between the NPVs calculated by using (1) the selling price guaranteed by the government $(\$ 27,845,376.65)$, and (2) the proposed agent-based model $(\$ 66,475,096.17)$. These results were presented to the experts (the 30 investors and 10 brokers who identified the risk factors and their impact) and their unanimous opinion was that the proposed model for determining the NPV is more realistic than the other method. The major strengths are identified as:

1. The NPV calculated using the guaranteed price is pessimistic and not realistic as it ignores the energy market dynamics. MAS accounts for potential changes in the market and in energy prices in different scenarios.

2. MAS accounts for the negotiations that would be carried out in different scenarios. Thus, both the impact of risks and how these risks change the negotiations between parties are taken into account.

However, the investors also point out that the MAS model does not provide any guidance on how the market can change in the future. There are no probabilities attached to the identified scenarios. The calculated NPV depends on the assumption that all of the 32 scenarios have the same probability of occurrence, which may not be a realistic assumption. The probability of occurrence of each and every scenario can be incorporated into the proposed MAS if expert opinion is elicited. Then, the NPV can be calculated considering the most likely, the mean (expected value), and the least likely scenarios.

This study was carried out in the period 2013-2014 and the model was finalized in December 2014. The energy prices prevailing in the Turkish energy market in 20092017 are shown in Figure 5. Taking the benchmark date as December 2014, it is clear that, if an investor tries to estimate the energy price for the forthcoming years based on prices between July 2009 and November 2014, the minimum price is $6.01 \mathrm{~d} / \mathrm{kWh}$ and the maximum price is $13.78 \$ / \mathrm{kWh}$ with an average price of $10.52 \$ / \mathrm{kWh}$. However, starting from 2015, there have been major changes in the market due to changes in the laws. The government guarantee has changed as the economic conditions have changed in Turkey. Indeed, between December 2014 and January 2018, the average price has been around $7 \mathrm{\Phi} / \mathrm{kWh}$, which is significantly lower than the prices before 2015 . The MAS model was able to predict this change in its worst case scenarios. Thus, it can be argued that the benefit of

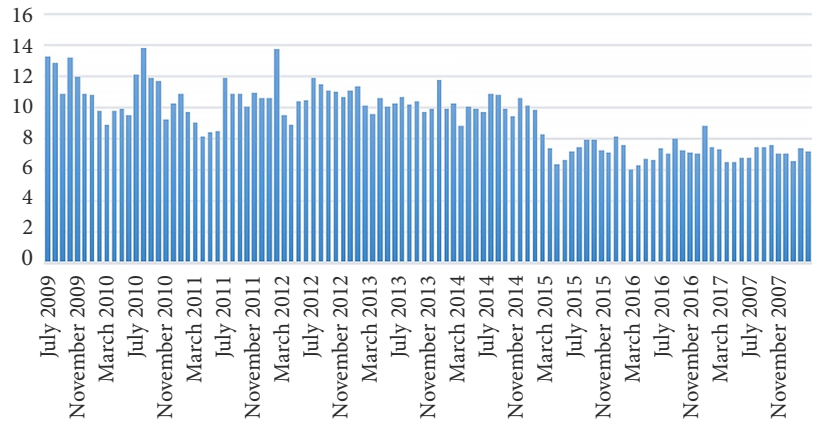

Figure 5. The energy prices prevailing in the Turkish energy market (EMMC, 2018)

the MAS model stems from its ability to consider alternative scenarios and also predict how the negotiations will change between the parties in these scenarios. It can also be argued that other probabilistic methods such as Monte Carlo Simulation can predict energy price under various scenarios, but the power of MAS stems from simulation of negotiations between parties which cannot be simulated in Monte Carlo Simulation. More discussions about the utilization of Monte Carlo Simulation for the prediction of energy prices can be found in Akcay et al. (2017).

\section{Conclusions}

The risk factors that impact the selling price of hydroelectric energy and negotiation between investors and brokers were determined in this study by surveying 10 brokers and 30 investors actively involved in the Turkish energy market. Then, all possible negotiation scenarios between investors and brokers were identified, and modelled by using a multi-agent system, using the Zeuthen Strategy. It can be observed that the energy prices predicted by MAS are quite realistic when compared to the actual energy prices in the years 2014-2018 in Turkey. The findings demonstrate that the currently performed NPV calculation that uses the price guaranteed by the government is pessimistic and may result in the rejection of a project that is actually profitable. This method is not realistic as it is based on the worst case scenario of selling at the price guaranteed by the government. The dynamics of the negotiation between the parties is based on the conditions prevailing in the energy market and is part and parcel of the proposed MAS, while these considerations are usually ignored in other methods such as Monte Carlo Simulation.

The significance of this study originates from the potential benefits to the hydropower industry, including the following:

- This is the first study in the literature that proposes a method that combines risk assessment and the negotiation process in determining the feasibility of a hydropower investment. A case study conducted at a hydropower plant project in Turkey tendered on a BOT basis provides evidence that the proposed method works well. 
- Using the proposed method, investors can carry out realistic feasibility studies. Investors can use this method to calculate their NPV and prepare realistic offers when negotiating with brokers. If bank credits are used for the investment, which is usually the case, investor can make more realistic income predictions and schedule the repayment of bank credit more realistically.

- Brokers can estimate the buying price of energy under different scenarios. Brokers buy energy from investors and sell this energy to users such as factories, hospitals, and hotels. Their profit depends on the difference between the selling price of the energy to the users and the buying price of the energy from the investors. Using the proposed method, brokers can make a more realistic estimate of the price of energy, and consequently can maximize their profit.

It should be noted however that the predictive capability of the MAS model can be as good as the assumptions made. If unexpected scenarios (black swans or perfect storms) actually happen, the proposed MAS model cannot reflect the selling price of energy under such unexpected conditions. Similarly, the dynamics of the market may change due to new legislation changing the negotiation rules/protocols between the parties completely. However, it is believed that this study demonstrates how a MAS can be structured considering the current practice of the negotiation process and the assumptions about risks in building scenarios. The generic framework can still be used by adjusting the risks used in building scenarios, and by incorporating new negotiation protocols specified by potential users in the future.

Finally, the MAS model proposed in this study can be applied to a variety of cases where uncertainty is high, and incomes and costs are realized as a result of negotiations between different parties. Projects tendered on a BOT basis are potential candidates for the application of this method as uncertainties are high and the incomes are prone to different factors such as demand and negotiations with the government. It is believed that the method proposed in this study can also generate realistic results in transportation projects such as toll roads.

\section{Disclosure statement}

We don't have any competing financial, professional, or personal interests from other parties.

\section{References}

Akcay, E. C., Dikmen, I., Birgonul, M. T., \& Arditi, D. (2017). Estimating the profitability of hydropower investments with a case study from Turkey. Journal of Civil Engineering and Management, 23(8), 1002-1012. https://doi.org/10.3846/1392 3730.2017.1350877

Cleary, P. J. (2001). The negotiation handbook. M. E. Sharpe, Inc. De Oliveira, E., Fonseca, J., \& Steiger-Garcao, A. (1997). MACIV: A DAI based resource management system. Applied Artificial Intelligence, 11(6), 525-550.

https://doi.org/10.1080/088395197118055
Du, J., \& El-Gafy, M. (2012). Virtual organizational imitation for construction enterprises: Agent-based simulation framework for exploring human and organizational implications in construction management. Journal of Computing in Civil Engineering, 26(3), 282-297.

https://doi.org/10.1061/(ASCE)CP.1943-5487.0000122

El-Adaway, I., \& Kandil, A. (2010). Multi-agent system for construction dispute resolution (MAS-COR). Journal of Construction Engineering and Management, 136(3), 303-315. https://doi.org/10.1061/(ASCE)CO.1943-7862.0000144

EMMC. (2018). Energy market management company. Retrieved from https://www.epias.com.tr/en

Farshchian, M. M., Heravi, G., \& AbouRizk, S. (2017). Optimizing the owner's scenarios for budget allocation in a portfolio of projects using agent-based simulation. Journal of Construction Engineering and Management, 143(7). https://doi.org/10.1061/(ASCE)CO.1943-7862.0001315

González-Briones, A., Chamoso, P., De La Prieta, F., Demazeau, Y., \& Corchado, J. M. (2018). Agreement technologies for energy optimization at home. Sensors, 18(5), 1633. https://doi.org/10.3390/s18051633

González-Briones, A., Prieto, J., De La Prieta, F., Herrera-Viedma, E., \& Corchado, J. M. (2018). Energy optimization using a case-based reasoning strategy. Sensors, 18(3), 865. https://doi.org/10.3390/s18030865

Karakas, K. (2010). Development of a multi agent system for negotiation of cost overrun in international construction projects (MSc thesis). Middle East Technical University.

Karakas, K., Dikmen I., \& Birgonul, M. T. (2013). Multiagent system to simulate risk-allocation and cost-sharing processes in construction projects. Journal of Computing in Civil Engineering, 27(3), 307-319.

https://doi.org/10.1061/(ASCE)CP.1943-5487.0000218

Kim, K., \& Paulson, B. (2003). Agent-based compensatory negotiation methodology to facilitate distributed coordination of project schedule changes. Journal of Computing in Civil Engineering, 17(1), 10-18. https://doi.org/10.1061/(ASCE)0887-3801(2003)17:1(10)

Kraus, S., Wilkenfeld, J., \& Zlotkin, G. (1995). Multiagent negotiation under time constraints. Artificial Intelligence, 75(2), 297-345. https://doi.org/10.1016/0004-3702(94)00021-R

Molinero, C., \& Núñez, M. (2011). Planning of work schedules through the use of a hierarchical multi-agent system. Automation in Construction, 20(8), 1227-1241.

https://doi.org/10.1016/j.autcon.2011.05.006

Mostafavi, A., Abraham, D., DeLaurentis, D., Sinfield, J., Kandil, A., \& Queiroz, C. (2015). Agent-based simulation model for assessment of financing scenarios in highway transportation infrastructure systems. Journal of Computing in Civil Engineering, 30(2).

https://doi.org/10.1061/(ASCE)CP.1943-5487.0000482

Ng, S. T., \& Li, W. (2006). Parallel bargaining protocol for automated sourcing of construction suppliers. Automation in Construction, 15(3), 365-373.

https://doi.org/10.1016/j.autcon.2005.07.004

Ozdoganm, I. D., \& Birgonul, M. T. (2000). A decision support framework for project sponsors in the planning stage of build-operate-transfer (BOT) projects. Construction Management and Economics, 18(3), 343-353. https://doi.org/10.1080/014461900370708

Ren, Z., \& Anumba, C. (2004). Multi-agent systems in construction - state of the art and prospects. Automation in Construction, 13(3), 421-434. https://doi.org/10.1016/j.autcon.2003.12.002 
Shen, L. Y., \& Wu, Y. Z. (2005). Risk concession model for build operate transfer contract projects. Journal of Construction Engineering Management, 131(2), 211-220.

https://doi.org/10.1061/(ASCE)0733-9364(2005)131:2(211)

Shoham, Y. (1993). Agent-oriented programming. Artificial Intelligence, 60(1), 51-92. https://doi.org/10.1016/0004-3702(93)90034-9

Song, J., Song, D., \& Zhang, D. (2015). Modeling the concession period and subsidy for BOT waste-to-energy incineration projects. Journal of Construction Engineering Management, 141(10).

https://doi.org/10.1061/(ASCE)CO.1943-7862.0001005

Taghaddos, H., Hermann, U., AbouRizk, S., \& Mohamed, Y. (2014). Simulation-based multiagent approach for scheduling modular construction. Journal of Computing in Civil Engineering, 28(2).

https://doi.org/10.1061/(ASCE)CP.1943-5487.0000262

Tah, J. H. (2005). Towards an agent-based construction supply network. Automation in Construction, 14(3), 353-359. https://doi.org/10.1016/j.autcon.2004.08.003

Taylor, J. E., Levitt, R., \& Villarroel, J. A. (2009). Simulating learning dynamics in project networks. Journal of Construction Engineering and Management, 135(10), 1009-1015. https://doi.org/10.1061/(ASCE)CO.1943-7862.0000065
Turkish Contractors Association. (2017). Retrieved from https:// www.tmb.org.tr/

Xue, X., Li, X., Shen, Q., \& Wang, Y. (2005). An agent-based framework for supply chain coordination in construction. Automation in Construction, 14(3), 413-430. https://doi.org/10.1016/j.autcon.2004.08.010

Xue, X. L., Shen, Q. P., O'Brien, W., \& Ren, Z. M. (2009). Improving agent-based negotiation efficiency in construction supply chains: A relative entropy method. Automation in Construction, 18(7), 975-982. https://doi.org/10.1016/j.autcon.2009.05.002

Zayed, T., \& Chang, L. (2002). Prototype model for build-operate-transfer risk assessment. Journal of Management in Engineering, 18(1), 7-16.

https://doi.org/10.1061/(ASCE)0742-597X(2002)18:1(7)

Zhu, L., Zhao, X., \& Chua, D. K. H. (2016). Agent-based debt terms' bargaining model to improve negotiation inefficiency in PPP projects. Journal of Computing in Civil Engineering, 30(6). https://doi.org/10.1061/(ASCE)CP.1943-5487.0000571 